University of Nebraska - Lincoln

DigitalCommons@University of Nebraska - Lincoln

$10-2011$

\title{
Two-photon double ionization of helium: Evolution of the joint angular distribution with photon energy and two-electron energy sharing
}

\author{
Zheng Zhang \\ Peking University \\ Liang-You Peng \\ Department of Physics and State Key Laboratory for Mesoscopic Physics, Peking University, Beijing, \\ liangyou.peng@pku.edu.cn \\ Ming-Hui Xu \\ Peking University \\ Anthony F. Starace \\ University of Nebraska-Lincoln, astarace1@unl.edu \\ Toru Morishita \\ University of Electro-Communications, Tokyo \\ See next page for additional authors
}

Follow this and additional works at: https://digitalcommons.unl.edu/physicsstarace

Part of the Physics Commons

Zhang, Zheng; Peng, Liang-You; Xu, Ming-Hui; Starace, Anthony F.; Morishita, Toru; and Gong, Qihuang, "Two-photon double ionization of helium: Evolution of the joint angular distribution with photon energy and two-electron energy sharing" (2011). Anthony F. Starace Publications. 186.

https://digitalcommons.unl.edu/physicsstarace/186

This Article is brought to you for free and open access by the Research Papers in Physics and Astronomy at DigitalCommons@University of Nebraska - Lincoln. It has been accepted for inclusion in Anthony F. Starace Publications by an authorized administrator of DigitalCommons@University of Nebraska - Lincoln. 


\section{Authors}

Zheng Zhang, Liang-You Peng, Ming-Hui Xu, Anthony F. Starace, Toru Morishita, and Qihuang Gong 


\title{
Two-photon double ionization of helium: Evolution of the joint angular distribution with photon energy and two-electron energy sharing
}

\author{
Zheng Zhang, ${ }^{1}$ Liang-You Peng, ${ }^{1,}{ }^{*}$ Ming-Hui Xu, ${ }^{1}$ Anthony F. Starace, ${ }^{2}$ Toru Morishita, ${ }^{3}$ and Qihuang Gong ${ }^{1, \dagger}$ \\ ${ }^{1}$ State Key Laboratory for Mesoscopic Physics and Department of Physics, Peking University, Beijing 100871, China \\ ${ }^{2}$ Department of Physics and Astronomy, The University of Nebraska, Lincoln, Nebraska 68588-0299, USA \\ ${ }^{3}$ Department of Engineering Science, University of Electro-Communications, 1-5-1 Chofu-ga-oka, Chofu-shi, Tokyo 182-8585, Japan
}

(Received 22 July 2011; published 6 October 2011)

\begin{abstract}
$A b$ initio calculations of two-photon double ionization of helium with photon energies varying from the nonsequential regime to well above the double-ionization threshold are presented. A systematic study of the joint angular distributions of the two ionized electrons at different energy sharing shows that the role of electron correlations is imprinted in the joint angular distribution. In particular, a rather general pattern is identified in the nonsequential regime that is independent of photon energy, pulse length, and energy sharing between the two electrons. Interestingly, the same distribution pattern is found for the equal-energy-sharing case, even when the photon energy is well above the double-ionization threshold. In the case of an extremely uneven energy sharing, the distribution pattern changes drastically as the photon energy is increased. In particular, when the photon energy is greater than the second-ionization threshold, the dominant emission mode of the two electrons switches gradually from "back to back" to "side by side." Finally, the joint angular distribution is found to provide clear evidence of the role of electron correlations in the initial state.
\end{abstract}

DOI: 10.1103/PhysRevA.84.043409

PACS number(s): $32.80 . \mathrm{Rm}, 32.80 . \mathrm{Wr}$

\section{INTRODUCTION}

Continued advances in laser technologies have enabled scientists to probe and even control the fundamental dynamics of electron correlations. As the simplest and most fundamental two-electron system, helium provides an ideal venue for exploring electron correlation dynamics. In the early 1990s, experiments provided evidence of nonsequential double ionization (NSDI) of helium by simultaneous absorption of many photons from intense laser fields [1,2]. Electron correlations play a necessary and unique role in NSDI, which cannot be explained within an independent electron model. Later, more detailed experimental measurements gave the differential momentum distributions of the two ionized electrons [3-5]. These experiments found pronounced signatures of correlated electron motion that disappear for high laser intensities [3]. Under high resolution, these signatures were found to include "fingerlike" [4] or "V-shaped" [5] structures. The joint momentum distributions were also shown to be sensitive to the carrier envelope phase (CEP) of a few-cycle laser pulse [6,7]. More recent experiments on double ionization of atoms using femtosecond laser pulses continue to reveal interesting phenomena, such as the anticorrelation of the two-electron momenta $[8,9]$.

In recent years, the rapid development of free-electron laser (FEL) technologies has enabled some important FEL facilities to provide ultrashort xuv pulses at rather high intensities $\left(>10^{14} \mathrm{~W} / \mathrm{cm}^{2}\right)$ [10-12]. Simultaneously, attosecond light sources using high-order harmonic generation have produced even shorter pulses at a number of different photon energies, including even single-cycle attosecond pulses [13,14]. Owing to the increasing availability of attosecond pulses and the promise of near-future increases in their intensities as well

\footnotetext{
*liangyou.peng@pku.edu.cn

†qhgong@pku.edu.cn
}

as the near-future promise of even subfemtosecond FEL light pulses, it is now becoming feasible to study electron correlation dynamics on its natural time scale $[15,16]$. Moreover, using the ultrashort pulses of these xuv laser sources, truly direct multiple excitation or ionization of several electrons bound in atoms or molecules becomes possible [17]. This contrasts with the case of intense femtosecond lasers, in which multiple ionization or excitation is achieved through the recollision mechanism $[1,18]$.

A typical few-photon process involving atoms and molecules in xuv fields is two-photon double ionization (TPDI) of helium, which has attracted considerable attention. Indeed, it has become a subfield of its own, with many theoretical investigations $[15,16,19-49]$ and several experimental studies [50-53]. In the long-pulse-duration limit, TPDI of helium can be classified into two types: "sequential" and "nonsequential." In the former, which is dominant when the photon energy is larger than the second-ionization threshold $(54.4 \mathrm{eV})$, the two electrons each absorb a single photon independently and are ionized sequentially. In this case, double ionization need not involve electron correlation. However, when the photon energy is smaller than the second-ionization threshold energy but larger than half the double-ionization threshold energy $(39.5 \mathrm{eV})$, double ionization can only proceed by means of electron correlation. Despite many different theoretical investigations, a number of challenges remain to be resolved. For example, results for even the total cross section for two-photon double ionization in the nonsequential regime have not yet achieved complete agreement with each other (cf. Figs. 1 and 2 of Ref. [47]). In particular, when the photon energy approaches $54.4 \mathrm{eV}$, the differences become larger. These facts imply that the full-dimensional computation of TPDI of He is indeed a very challenging and demanding task. At the same time, due to the difficulties and uncertainties of the very few experimental measurements, the measured data cannot determine which are the better calculations. 
Most previous studies have focused on calculating the total cross section for TPDI in the nonsequential regime and on laser-pulse-duration effects. However, increasingly, investigations of TPDI treat various kinds of angular distributions. By far the majority of these latter studies fix the emission direction (angle) of one electron and present the relative angular distribution of the other electron [16,27,30,36,38,39,43,47]. Others present polar plots of the angular distribution of one electron resulting from integration of the triply differential cross section (TDCS) over all emission angles of the other electron $[29,41,49]$. Still others present nuclear recoil angular distributions [40,45,47]. As far as we know, the TDCS as a function of the emission angles of both ionized electrons [which we call here the joint angular distribution (JAD)] still needs to be thoroughly investigated. The behavior of this JAD as a function of the photon energy and the energy sharing between the two ionized electrons is the subject of this work.

A number of features of the TDCS for the TPDI process have been learned. The consensus is that the two ionized electrons are highly correlated, and that they prefer being ejected back to back. When the photon energy approaches $39.5 \mathrm{eV}$, the energy spectrum of the electrons is quite flat, indicating that both equal and very unequal energy sharing have comparable probability. For photon energies approaching $54.4 \mathrm{eV}$, the total cross section increases sharply, and the two electrons tend to share the excess energy unequally. When the photon energy is larger than $54.4 \mathrm{eV}$, uncorrelated sequential ionization dominates. However, the existence of an "anomalous component" at equal energy sharing has been found [25,28,29], which suggests that correlation effects cannot be completely neglected. Moreover, according to the perturbative model of Ref. [41], a deviation from the dipole angular distribution of one electron (when the TDCS is integrated over all emission angles of the other electron) is found, not only at equal energy sharing but also for highly unequal energy sharing.

Nevertheless, a number of features of the TPDI process are still not completely understood. These include the dependence of electron correlation effects on the energy sharing, especially in the case of extreme energy sharing. Also, a detailed comparison of correlation effects in the sequential regime with those in the nonsequential regime is still needed, both to deepen our understanding of the origin of various correlation mechanisms and to understand their characteristic time scales.

In this work, by accurately solving the full-dimensional, two-electron time-dependent Schrödinger equation, we investigate theoretically the TPDI process in both the nonsequential and the sequential regime. In particular, we analyze the JAD of the two ionized electrons for different energy sharing and different photon energies. Our motivation is to identify the role of electron correlation effects in the three different ionization stages [46]; that is, the exchange of energy between the two electrons in the initial, intermediate, and final states. The initial and final states refer to times before and after the interaction with the laser pulse. The intermediate state refers to the energy exchange between the electrons (owing to the Coulomb interaction) during their interaction with the laser pulse [54]. In order to discover whether electron correlations play a role in the sequential ionization region, we have carried out a detailed study of the JAD for TPDI for photon energies larger than the second-ionization threshold and for different electron energy sharing. By comparing these results with those for the nonsequential double-ionization regime, we find that electron correlations in the intermediate state are important for the equal-energy-sharing case, regardless of the photon energy. On the contrary, in the case of extremely unequal energy sharing, electron correlations in the final state are found to become dominant as the photon energy increases.

The rest of this paper is organized as follows: In Sec. II, we briefly describe our theoretical methods for the numerical solution of the full-dimensional time-dependent Schrödinger equation (TDSE) for the two electrons of a He atom interacting with a laser field. In Sec. III, we first present comparisons of our results with those in some previous works in order to demonstrate the accuracy of our numerical methods. We then present a detailed analysis of the JADs of the two ionized electrons for different photon energies and different energysharing apportionments. Finally, we present our conclusions in Sec. IV. Unless otherwise stated, atomic units (a.u.) are used throughout this work.

\section{THEORETICAL METHODS}

In this section we present a brief account of our theoretical methods for treating the process of two-photon double ionization of helium. Our main approach is to numerically solve the corresponding TDSE of the two-electron system interacting with a short laser pulse. After the end of the laser pulse, the final-state wave function is further propagated freely for a sufficiently long time into the asymptotic region. The differential and total-ionization cross sections are then extracted by projecting the wave function onto the product of two Coulomb waves with $Z=2$. We note that this method for extracting cross sections from the numerical wave functions in the asymptotic region is quite accurate and reliable, as has been shown in the detailed analyses and careful numerical verifications presented in Refs. [38,55].

\section{A. The close-coupling scheme}

We employ spherical coordinates so as to use the usual close-coupling scheme to treat the angular coordinates analytically. The TDSE of helium in a linearly polarized laser field is given by

$$
i \frac{\partial}{\partial t} \Psi\left(\mathbf{r}_{1}, \mathbf{r}_{2}, t\right)=H(t) \Psi\left(\mathbf{r}_{1}, \mathbf{r}_{2}, t\right),
$$

where the Hamiltonian operator, in the dipole approximation (length gauge), can be written as

$$
\begin{aligned}
H\left(\mathbf{r}_{1}, \mathbf{r}_{2}, t\right)= & \frac{\mathbf{p}_{1}^{2}}{2}+\frac{\mathbf{p}_{2}^{2}}{2}-\frac{2}{r_{1}}-\frac{2}{r_{2}}+\frac{1}{\left|\mathbf{r}_{1}-\mathbf{r}_{2}\right|} \\
& +\left(\mathbf{r}_{1}+\mathbf{r}_{2}\right) \cdot \mathbf{E}(t),
\end{aligned}
$$

where $\mathbf{E}(t)$ is the electric field of the laser pulse.

In the close-coupling scheme, the two-electron wave function $\Psi\left(\mathbf{r}_{1}, \mathbf{r}_{2}, t\right)$ is expanded in coupled spherical harmonics,

$$
\Psi\left(\mathbf{r}_{1}, \mathbf{r}_{2}, t\right)=\sum_{L, M, l_{1}, l_{2}} \frac{R_{l_{1}, l_{2}}^{L, M}\left(r_{1}, r_{2}, t\right)}{r_{1} r_{2}} Y_{l_{1}, l_{2}}^{L, M}\left(\hat{\boldsymbol{r}}_{\mathbf{1}}, \hat{\boldsymbol{r}}_{\mathbf{2}}\right),
$$


in which

$$
\begin{aligned}
& Y_{l_{1}, l_{2}}^{L, M}\left(\hat{r}_{1}, \hat{r}_{2}\right) \\
& \quad=\sum_{m_{1}, m_{2}}\left\langle l_{1} m_{1} l_{2} m_{2} \mid l_{1} l_{2} L M\right\rangle Y_{l_{1}, m_{1}}\left(\hat{\boldsymbol{r}}_{1}\right) Y_{l_{2}, m_{2}}\left(\hat{\boldsymbol{r}}_{2}\right),
\end{aligned}
$$

where $\left\langle l_{1} m_{1} l_{2} m_{2} \mid l_{1} l_{2} L M\right\rangle$ is the usual Clebsch-Gordan coefficient.

In this work, the laser pulse is assumed to be linearly polarized and the two electrons are assumed to be initially in their ground state. Therefore, the total magnetic quantum number $M$ in Eq. (3) is conserved and equal to zero. Substituting Eq. (3) into Eq. (1), one obtains the following set of coupled differential equations:

$$
\begin{aligned}
& i \frac{\partial}{\partial t} \frac{R_{l_{1}, l_{2}}^{L}\left(r_{1}, r_{2}, t\right)}{r_{1} r_{2}} \\
& \quad=\sum_{L^{\prime}, l_{1}^{\prime}, l_{2}^{\prime}}\left\langle l_{1}, l_{2}, L, 0|H| l_{1}^{\prime}, l_{2}^{\prime}, L^{\prime}, 0\right\rangle \frac{R_{l_{1}^{\prime}, l_{2}^{\prime}}^{L^{\prime}}\left(r_{1}, r_{2}, t\right)}{r_{1} r_{2}} .
\end{aligned}
$$

Owing to the fact that the two electrons are initially in a singlet spin state (and since we employ $L S$ coupling), $\Psi\left(\mathbf{r}_{1}, \mathbf{r}_{2}, t\right)=$ $\Psi\left(\mathbf{r}_{2}, \mathbf{r}_{1}, t\right)$. The radial part of the wave function must thus satisfy the following relation:

$$
R_{l_{1}, l_{2}}^{L}\left(r_{1}, r_{2}, t\right)=(-1)^{l_{1}+l_{2}-L} R_{l_{2}, l_{1}}^{L}\left(r_{2}, r_{1}, t\right) .
$$

In practical computations, one thus includes only those partial waves having an even integer value for $l_{1}+l_{2}-L$, which greatly reduces the total number of partial waves. In addition, this symmetry allows one to restrict the computations to only the upper triangle of $R_{l_{1}, l_{2}}^{L}\left(r_{1}, r_{2}, t\right)$ with $r_{1} \geqslant r_{2}$. Taking advantage of these symmetries, one can greatly reduce the storage and computation times.

\section{B. Discretization of radial coordinates and choice of time propagator}

For the discretization of the radial wave function $R_{l_{1}, l_{2}}^{L}\left(r_{1}, r_{2}, t\right)$, we employ the finite element discrete variable representation (FE-DVR), which has been successfully applied in numerous studies of the dynamics of few-electron atomic and molecular systems in intense laser fields (see, e.g., [38-40,56-58]). For details of the FE-DVR, please consult these previous works. Here, we only give a very brief account of the essence of this method.

In the FE-DVR method, the configuration space is divided into many different finite elements within which the wave function is further expanded using low-order Gauss-Lobatto DVR basis functions. Two neighboring elements are connected by introducing a "bridge" function to ensure continuity [56]. According to previous work by others and our own experiences, the FE-DVR method has at least three prominent advantages: First, it provides a sparse matrix representation of the kinetic energy operator and a simple diagonal representation of the potentials. Second, the length of each finite element is independently adjustable. One can thus adopt relatively dense mesh points to describe the potential near the atomic core and relatively sparse mesh grids at larger distances. Both the accuracy and efficiency can be simultaneously ensured. Finally, we find that this method requires only very little data communication between adjacent CPUs when one programs the algorithm with the message-passing interface (MPI). Thus, the FE-DVR method is very suitable for parallel computation.

For a good representation of Coulomb repulsion between the two electrons, $1 /\left|\mathbf{r}_{1}-\mathbf{r}_{2}\right|$, we follow the treatment of McCurdy and coworkers [59] by solving a Poisson equation. We find this method is both efficient and accurate.

Temporal propagation of the wave function is carried out using the Arnoldi propagator, whose accuracy and stability have already been verified in our previous works $[46,60,61]$. This propagator has also been widely used by many other groups (see, e.g., Refs. [38,39] and references therein).

\section{Extraction of ionization cross section}

After the end of the laser pulse, the ionization cross section has to be calculated from the resultant wave function. However, due to the well-known difficulty in representing the field-free double continuum including electron correlation effects, it is a nontrivial job to extract the exact triply differential and totalionization cross sections [62]. Usually, one must use some approximate representation. We have adopted the following procedure (which has been employed by several other groups $[16,27,38,39,43,55])$ : we further propagate the wave function freely for a sufficiently long time after the end of the laser pulse so that we approach the asymptotic region (in which the large distance between the two ionized electrons makes their Coulomb repulsion negligible). In this case, it is reasonable to approximate the real double continuum by the uncorrelated double continuum (i.e., the product of two Coulomb waves with $Z=2$ ). The detailed analyses and quantitative comparisons in Refs. [38,55] show that the two-electron wave packet quickly enters the asymptotic region. Therefore, the projection of our further-propagated two-electron wave function onto the product of two Coulomb waves is able to give a reasonably accurate result for the differential ionization cross section [38]. Actually, based on exterior complex scaling (ECS) of the electronic coordinates, Palacios et al. [44] formulated a different method to extract the triply differential cross section for double ionization of helium. Their method took all electronic interactions fully into account, including the Coulomb repulsion of the two electrons. Their results agree very well with those of Feist et al. [38], with the same shapes but slightly larger peak amplitudes (cf. Fig. 9 of Ref. [44]).

A one-electron Coulomb wave function satisfying the incoming-wave boundary condition has the form [63]

$$
\phi_{\mathbf{k}}^{(-)}(\mathbf{r})=\frac{1}{\sqrt{k}} \sum_{l m} i^{l} e^{-i\left[\sigma_{l}(k)+\delta_{l}(k)\right]} Y_{l m}(\hat{\boldsymbol{r}}) Y_{l m}^{*}(\hat{\boldsymbol{k}}) R_{E l}(r),
$$

where $\sigma_{l}(k)=\arg \Gamma(l+1+i \eta)$ is the Coulomb phase shift, $\eta=-(Z-N) / k, Z$ is the nuclear charge, $N$ is the number of electrons remaining in the ion, and $\delta_{l}(k)$ is the $l$ th partial wave phase shift (with respect to Coulomb waves) due to any nonCoulomb short-range part of the potential. For a hydrogenic atom, $\delta_{l}=0$. The radial wave function $R_{k l}$ has a momentum normalization,

$$
\int R_{k l}(r) R_{k^{\prime} l}(r) r^{2} d r=2 \pi \delta\left(k-k^{\prime}\right),
$$

while $R_{E l}=\frac{1}{\sqrt{2 \pi k}} R_{k l}$ is energy normalized. 
The density distribution, $P\left(k_{1}, \hat{k}_{1}, k_{2}, \hat{k}_{2}\right)$, of the two ionized electrons in momentum space is obtained by projecting the final-state wave function onto the uncorrelated doublecontinuum state $\phi_{\mathbf{k}_{1}}^{(-)}\left(\mathbf{r}_{1}\right) \phi_{\mathbf{k}_{2}}^{(-)}\left(\mathbf{r}_{2}\right)$; that is,

$$
\begin{array}{r}
P\left(k_{1}, \hat{k}_{1}, k_{2}, \hat{k}_{2}\right)= \\
=\frac{\left.\left|\left\langle\phi_{\mathbf{k}_{1}}^{(-)}\left(\mathbf{r}_{1}\right) \phi_{\mathbf{k}_{2}}^{(-)}\left(\mathbf{r}_{2}\right)\right| \Psi\left(\mathbf{r}_{1}, \mathbf{r}_{2}, t_{f}\right)\right)\right|^{2}}{4 \pi^{2} k_{1}^{2} k_{2}^{2}} \mid \sum_{L, l_{1}, l_{2}}(-1)^{l_{1}+l_{2}} e^{i\left(\sigma_{l_{1}}+\sigma_{l_{2}}\right)} \\
\quad \times\left. Y_{l_{1}, l_{2}}^{L 0}\left(\hat{\boldsymbol{k}}_{\mathbf{1}}, \hat{\boldsymbol{k}}_{2}\right) M_{l_{1}, l_{2}}^{L}\left(k_{1}, k_{2}\right)\right|^{2},
\end{array}
$$

where

$M_{l_{1}, l_{2}}^{L}\left(k_{1}, k_{2}\right)=\int d r_{1} \int d r_{2} r_{1} r_{2} R_{k_{1} l_{1}}\left(r_{1}\right) R_{k_{2} l_{2}}\left(r_{2}\right) R_{l_{1}, l_{2}}^{L}\left(r_{1}, r_{2}, t_{f}\right)$.

The total $\mathrm{N}$-photon double-ionization cross section is then given by

$$
\sigma_{N}=\left(\frac{\omega}{I_{0}}\right)^{N} \frac{1}{T_{\text {eff }}} \int d^{3} \mathbf{k}_{1} \int d^{3} \mathbf{k}_{2} P\left(k_{1}, \hat{k}_{1}, k_{2}, \hat{k}_{2}\right),
$$

where the effective interaction time $T_{\text {eff }}[25,27,39]$ for an $N$ photon process is defined by

$$
T_{\text {eff }}=\int d t\left(\frac{I(t)}{I_{0}}\right)^{N},
$$

with $I(t)$ being the laser intensity profile and $I_{0}$ being the peak intensity.

In the present work, we assume the laser pulse has a $\sin ^{2}$ envelope. In this case, $T_{\text {eff }}$ equals $35 T / 128$, where $T$ is the duration of the laser pulse. Therefore, the total generalized cross section for TPDI takes the form

$$
\sigma=\frac{1}{4 \pi^{2}}\left(\frac{\omega}{I_{0}}\right)^{2} \frac{1}{T_{\text {eff }}} \int d k_{1} d k_{2} \sum_{L, l_{1}, l_{2}}\left|M_{l_{1}, l_{2}}^{L}\left(k_{1}, k_{2}\right)\right|^{2} .
$$

From this equation, the triply differential cross section (TDCS) can be derived through differentiation with respect to $E_{1}, \hat{\boldsymbol{k}}_{\mathbf{1}}$, and $\hat{\boldsymbol{k}}_{2}$ :

$$
\begin{aligned}
\frac{d^{3} \sigma}{d E_{1} d \hat{\boldsymbol{k}}_{\mathbf{1}} d \hat{\boldsymbol{k}}_{\mathbf{2}}} & =\frac{1}{k_{1}} \frac{d^{3} \sigma}{d k_{1} d \hat{\boldsymbol{k}}_{\mathbf{1}} d \hat{\boldsymbol{k}}_{\mathbf{2}}} \\
& =\left(\frac{\omega}{I_{0}}\right)^{2} \frac{1}{T_{\mathrm{eff}}} \int k_{2}^{2} d k_{2} k_{1} P\left(k_{1}, \hat{k}_{1}, k_{2}, \hat{k}_{2}\right) .
\end{aligned}
$$

The singly differential cross section (SDCS) can then be calculated by integrating Eq. (14) over $\hat{\boldsymbol{k}}_{\mathbf{1}}$ and $\hat{\boldsymbol{k}}_{\mathbf{2}}$,

$$
\frac{d \sigma}{d E_{1}}=\frac{1}{4 \pi^{2} k_{1}}\left(\frac{\omega}{I_{0}}\right)^{2} \frac{1}{T_{\text {eff }}} \int d k_{2} \sum_{L, l_{1}, l_{2}}\left|M_{l_{1}, l_{2}}^{L}\left(k_{1}, k_{2}\right)\right|^{2},
$$

giving the energy spectrum of one ionized electron.

Finally, we define $\varepsilon$ as the ratio of the kinetic energy of one electron to the total excess energy:

$$
\varepsilon=\frac{E_{i}}{E_{1}+E_{2}} \quad(i=1 \text { or } 2)
$$

Since the two ionized electrons are indistinguishable, their joint angular distribution (JAD) for the energy sharing $\varepsilon$ is calculated as follows:

$$
\begin{aligned}
P\left(\hat{k}_{1}, \hat{k}_{2} ; \varepsilon\right)= & \int k_{1}^{2} d k_{1} \int k_{2}^{2} d k_{2} \frac{1}{2}\left[\delta\left(\varepsilon-\frac{E_{2}}{E_{1}+E_{2}}\right)\right. \\
& \left.+\delta\left(\varepsilon-\frac{E_{1}}{E_{1}+E_{2}}\right)\right] P\left(k_{1}, \hat{k}_{1}, k_{2}, \hat{k}_{2}\right) .
\end{aligned}
$$

Owing to indistinguishability of the identical electrons, the JAD satisfies the relation $P\left(\hat{k}_{1}, \hat{k}_{2} ; \varepsilon\right)=P\left(\hat{k}_{1}, \hat{k}_{2} ; 1-\varepsilon\right)$. We shall use Eq. (17) to investigate the influence of the energy sharing (as specified by the parameter $\varepsilon$ ) on the JAD of the two ionized electrons.

Please note that $P\left(\hat{k}_{1}, \hat{k}_{2} ; \varepsilon\right)$ has five variables. In our discussions and corresponding graphical representations of $P\left(\hat{k}_{1}, \hat{k}_{2} ; \varepsilon\right)$, the azimuthal angles $\phi$ of the electron momenta are taken to be zero (i.e., $\phi_{1}=\phi_{2}=0$ ). Our graphs of the JADs for fixed energy sharing $\varepsilon$ thus present the following:

$$
P\left(\theta_{1}, \theta_{2} ; \varepsilon\right)=P\left(\theta_{1}, 0, \theta_{2}, 0 ; \varepsilon\right),
$$

where $\theta_{1}$ and $\theta_{2}$ are relative to the laser polarization axis.

\section{RESULTS AND DISCUSSION}

In this section, we focus first on demonstrating the reliability of our numerical methods and computer codes by presenting comparisons with prior results of others for various related properties of the helium atom. These comparisons concern the energies of the first few bound states, the total cross section for single-photon double ionization, and the differential cross section for two-photon nonsequential double ionization. We then present our results for the TPDI process, focusing on the joint angular distributions (JADs) of the two ionized electrons for photon energies in both the nonsequential and the sequential regime. Our goal is to identify the role played by electron correlation effects on the JADs. Our results indicate a quite general distribution pattern when electron correlations in the intermediate state become important in the ionization process.

\section{A. Validation of computational code}

As a first test of our code, we present in Table I some results for the energy eigenvalues of the first few bound states of He and compare them with the accurate tabulated values in Refs. [64,65]. We note that our FE-DVR grids are not optimized for a particular bound-state representation. Rather, in each case we employ a computationally simple evaluation of the entire Hamiltonian spectrum on a grid that provides a reasonable representation of many eigenstates simultaneously. Specifically, we have chosen the following parameters: $r_{1, \max }=r_{2, \max }=60$ a.u. with 64 finite elements and 8 basis functions in each element, $l_{1, \max }=l_{2, \max }=7$. The ground state is calculated using imaginary time propagation, in which case the TDSE turns into a diffusion equation. The higher bound states are similarly computed with recursive subtraction of lower states with the same symmetry from the trial wave function. During the computation of these eigenvalues, the total angular momentum number $L$ is taken to be 0,1 , and 2 for $S, P$, and $D$ states, respectively. The resultant 
TABLE I. Comparison of the energies (in a.u.) of the first few bound states of He calculated in the present work with results of Ref. [64] and the tabulation in Ref. [65], where $n L$ denotes the $n$th bound state having total angular momentum $L$.

\begin{tabular}{|c|c|c|c|c|c|c|}
\hline & $1 S$ & $2 S$ & $3 S$ & $4 S$ & $2 P$ & $3 D$ \\
\hline Present & -2.9036690 & -2.1459706 & -2.0612710 & -2.0335857 & -2.1238392 & -2.0555552 \\
\hline Ref. [64] & -2.9037244 & -2.1459740 & -2.0612720 & -2.0335877 & -2.1238431 & -2.0556207 \\
\hline Ref. [65] & -2.9037244 & -2.1459740 & -2.0612720 & -2.0335867 & -2.1238431 & -2.0556207 \\
\hline
\end{tabular}

eigenvalues are tabulated in Table I and agree with accurate results of others to 5 to 6 digits.

When He is exposed to photons with energy larger than the double-ionization threshold of $79 \mathrm{eV}$, it can be doubly ionized by absorbing a single photon owing to electron correlation effects. Before discussing TPDI, we consider first one-photon double ionization of $\mathrm{He}$, which has been thoroughly investigated in the literature both theoretically $[66,67]$ and experimentally $[68,69]$. In order to check the accuracy of our code for solving the two-electron TDSE, we calculate the total cross section for single-photon double ionization of $\mathrm{He}$ at the following five photon energies: 82, 92, 102, 110, and $125 \mathrm{eV}$. In Fig. 1, our numerical results are compared with smoothed data from accurate experimental measurements [69]. One sees that very good agreement is achieved. Total cross sections for even larger photon energies have also been calculated (not shown here) and the agreement with accurate experimental data gets even better.

We present now some tests of the accuracy of our code for the case of TPDI of He in the nonsequential regime. The TDCS provides a much more sensitive test of our code than does the total-ionization cross section, for which it is much easier to get a converged result [38]. We have chosen a photon energy of $42 \mathrm{eV}$ for these tests since, for this energy, TPDI has been intensively studied by various theoretical methods [27,33,38-40]. Although most previous results for the TDCS show qualitative agreement, the detailed shape and magnitude of the TDCS is very sensitive to the particular approximations

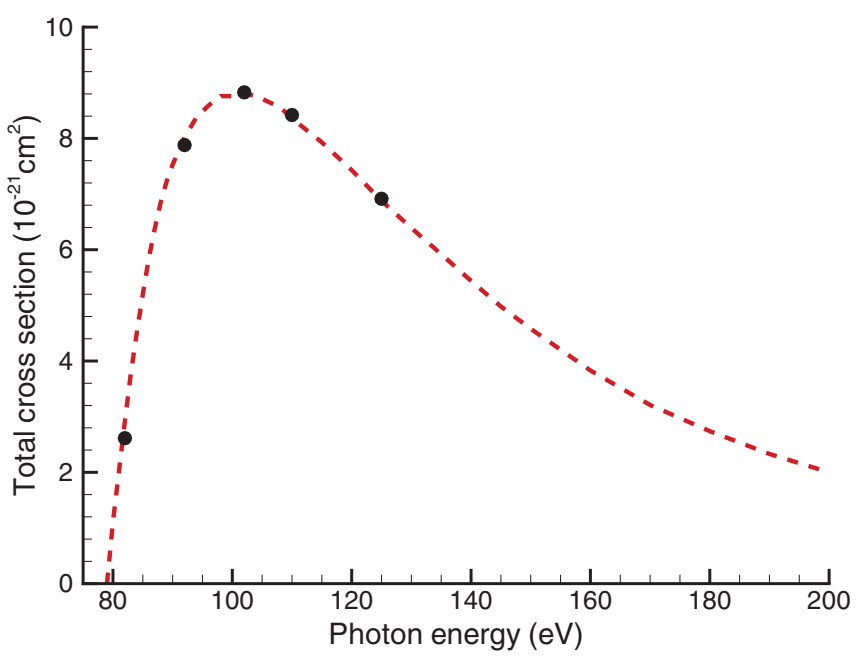

FIG. 1. (Color online) Comparison of the total one-photon double-ionization cross section of $\mathrm{He}$, calculated using the present approach for solving the two-electron TDSE (filled circles), with the experimental measurements (dashed line) of Ref. [69]. used to solve the TDSE and to the particular laser parameters chosen. Feist et al. [38] have carried out a very systematic and rigorous investigation of the accuracy of their results. In particular, they have checked carefully the convergence of their results for all chosen parameters and for approximating the final state by the product of two Coulomb waves. We note that comparisons of their TDCS results for a photon energy of $42 \mathrm{eV}$ with those of $\mathrm{Hu}$ et al. [27] and of Ivanov and Kheifets [33] are given in Fig. 10 of Ref. [38]. Moreover, Guan et al. [39] report that their TDCS results and those of Ref. [38] were found to be "very close." Finally, the $42 \mathrm{eV}$ photon energy TDCS results of Horner et al. [40] are compared with those of Feist et al. [38] in Fig. 5 of Ref. [40], showing excellent agreement for the energy positions of the zeros of the TDCS and moderate agreement for the magnitudes of the maxima. For these various reasons, we have chosen to demonstrate the accuracy of our TDCS results by comparing our results at $42 \mathrm{eV}$ with those of Feist et al. [38].

In principle, in order to extract the TDCS in the way described in Sec. II C, one should use a sufficiently long pulse with a low peak intensity. For this reason, we have chosen a 16-cycle $\sin ^{2}$ pulse with $I=1 \times 10^{12} \mathrm{~W} / \mathrm{cm}^{2}$. After the end of the laser pulse, the wave packets are propagated further for another 40 a.u. so that the projection onto the product of two Coulomb waves is valid. The box size is set to be 130 a.u. along each radial axis and 64 elements are used with 8 basis functions in each of them. The angular momenta numbers are set to be $\left(L_{\max }, l_{1, \max }, l_{2, \max }\right)=(2,7,7)$. The partial waves and box size employed here are not as large as those used in Ref. [38], but our results converge for these laser parameters. In Fig. 2, our TDSC [cf. Eq. (14)] at $E_{1}=2.5 \mathrm{eV}$ is shown as a function of the emission angle of the second electron $\theta_{2}$ for different ejection angles of the first electron $\theta_{1}$. The comparison in Fig. 2 of our results with those of Ref. [38] shows excellent qualitative agreement and good quantitative agreement, including for the most challenging case of $\theta_{1}=90^{\circ}$, which is the most unfavored emission direction.

We attribute the discrepancies in the magnitudes of the peak heights between our results and those of Ref. [38] (cf. Fig. 2) to the different pulse lengths used in the two calculations. Our results for a 16 -cycle $\sin ^{2}$ pulse are about $10 \% ;-20 \%$; lower than those of Ref. [38], in which a 40-cycle (about 4 fs) pulse was used. In order to rule out the possibility that these differences stem from numerical inaccuracies, we have carefully checked the convergence of our results for the spatial and temporal parameters we chose. In particular, we compared the results calculated at different times after the end of the laser pulse and find that the results are essentially the same for free propagation times of 30 and 

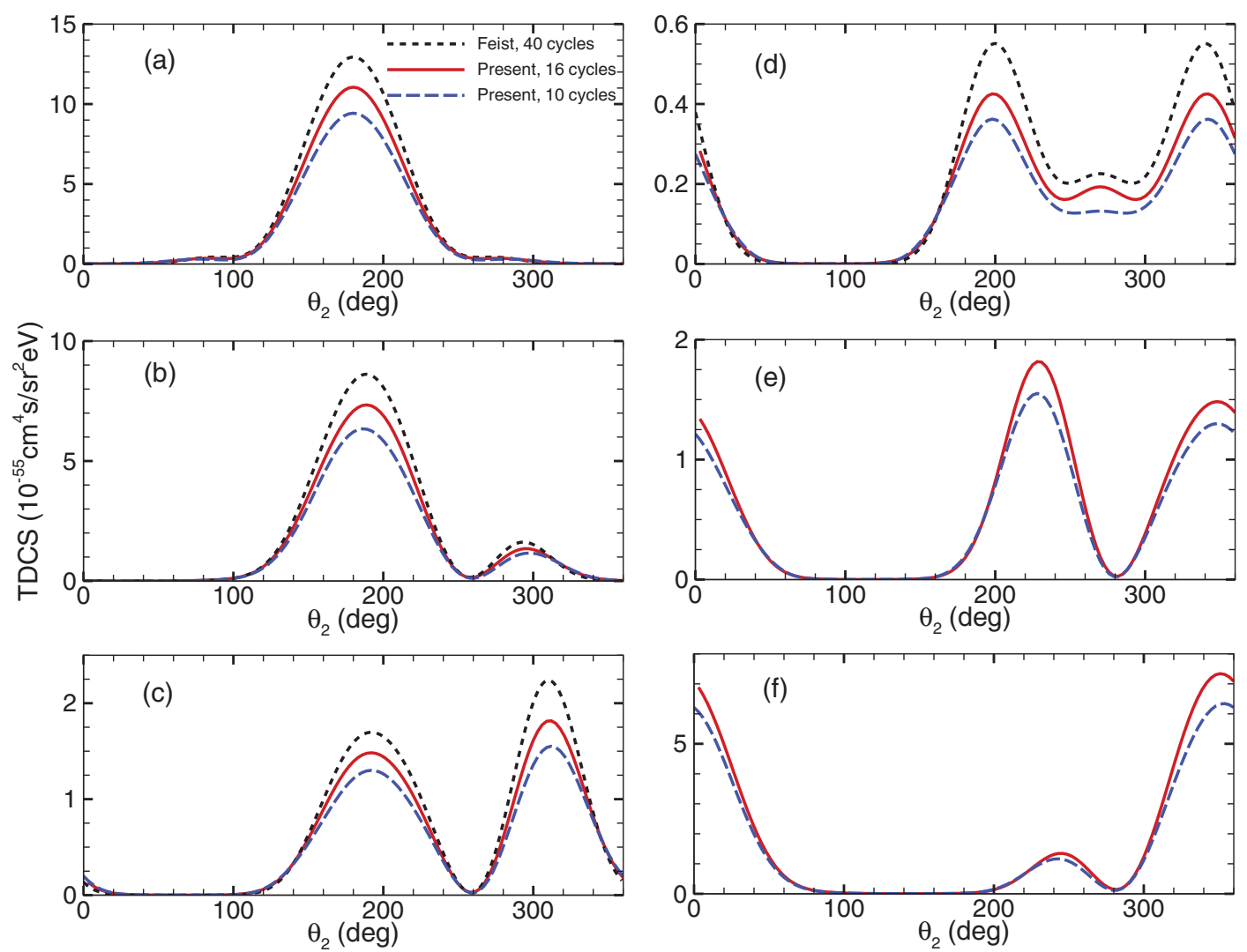

FIG. 2. (Color online) Triply differential cross section (TDCS) for two-photon double ionization of He plotted as a function of the ejection angle $\theta_{2}$ of the second electron (with respect to the laser polarization direction) for the photon energy $\omega=42 \mathrm{eV}$ and a first-electron kinetic energy $E_{1}=2.5 \mathrm{eV}$. The ejection angle of the first electron is fixed respectively at (a) $\theta_{1}=0^{\circ}$, (b) $\theta_{1}=30^{\circ}$, (c) $\theta_{1}=60^{\circ}$, (d) $\theta_{1}=90^{\circ}$, (e) $\theta_{1}=120^{\circ}$, and (f) $\theta_{1}=150^{\circ}$. Short-dashed lines are for Feist et al. [38] for a 40-cycle pulse, solid lines are for the present results for a 16-cycle pulse, and long-dashed lines are for the present results for a 10-cycle pulse.

40 a.u. In addition, we increased the maximum value of $L$ from 2 to 3 , and find that the differences are less than $1 \%$. Having thus eliminated numerical errors as the reason for the discrepancies, our attention turned next to the differing pulse lengths and spectral widths as the likely source of the discrepancies.

The full widths at half maximum (FWHM) of the spectral distributions are estimated to be $1.5 \mathrm{eV}$ and $3.75 \mathrm{eV}$, respectively, for 40- and 16-cycle pulses. The greater spectral width in our case results in a significant portion of the spectral intensity lying below the double-ionization threshold of $39.5 \mathrm{eV}$, which will inevitably lower the ionization probability. To further confirm that the discrepancies indeed come from the pulse length, we have done another calculation using a 10-cycle laser pulse, whose results are also shown in Fig. 2. As expected, the values of the TDCS are even lower. (The FWHM of the spectral distribution of the 10-cycle pulse is about $6 \mathrm{eV}$.) $\mathrm{We}$ also note that our results for the 10-cycle-pulse case are very close to those of Guan et al. (cf. Fig. 6 in [39]), although their results are calculated at the much higher intensity of $I=5 \times 10^{14} \mathrm{~W} / \mathrm{cm}^{2}$. However, in the perturbative regime, the pulse intensity should not matter. Unfortunately, treating the case of a 40-cycle pulse is beyond the capability of our computational resources. Nevertheless, we feel the tests we have done have pinned the source of the quantitative discrepancies to the pulse length (and associated spectral width) and that the present results for a 16-cycle pulse are highly accurate.

\section{B. Joint angular distribution for TPDI of He}

We turn our attention now to examining the effect of electron correlations in TPDI of He by systematically investigating the joint angular distributions (JADs) of the two ionized electrons at different photon energies and different energy sharings. For the results presented in the rest of this paper, the laser pulse is taken to have 16 optical cycles and a peak intensity $I_{0}=1 \times 10^{12} \mathrm{~W} / \mathrm{cm}^{2}$, unless otherwise specified. Using longer laser pulses does not significantly change the pattern of the JAD. Also, higher intensities up to $I_{0}=1 \times 10^{14} \mathrm{~W} / \mathrm{cm}^{2}$ have also been investigated, but no significant changes in the JAD pattern were found.

In Fig. 3, we show the JADs of the two ionized electrons for a photon energy of $42 \mathrm{eV}$ and four different energy sharings. The JAD is seen to be symmetric with respect to the two diagonal lines defined by $\theta_{2}=\theta_{1}$ and $\theta_{2}=-\theta_{1}+360^{\circ}$ (given by the white line in the figure). The JAD pattern at this photon energy is seen to be insensitive to the energy sharing: in each frame, there are four main peaks at the edges of the figures with four minor peaks along the line $\theta_{2}=-\theta_{1}+360^{\circ}$. The two electrons are thus most likely to be ejected in opposite directions (i.e., the so-called back-to-back emission), 

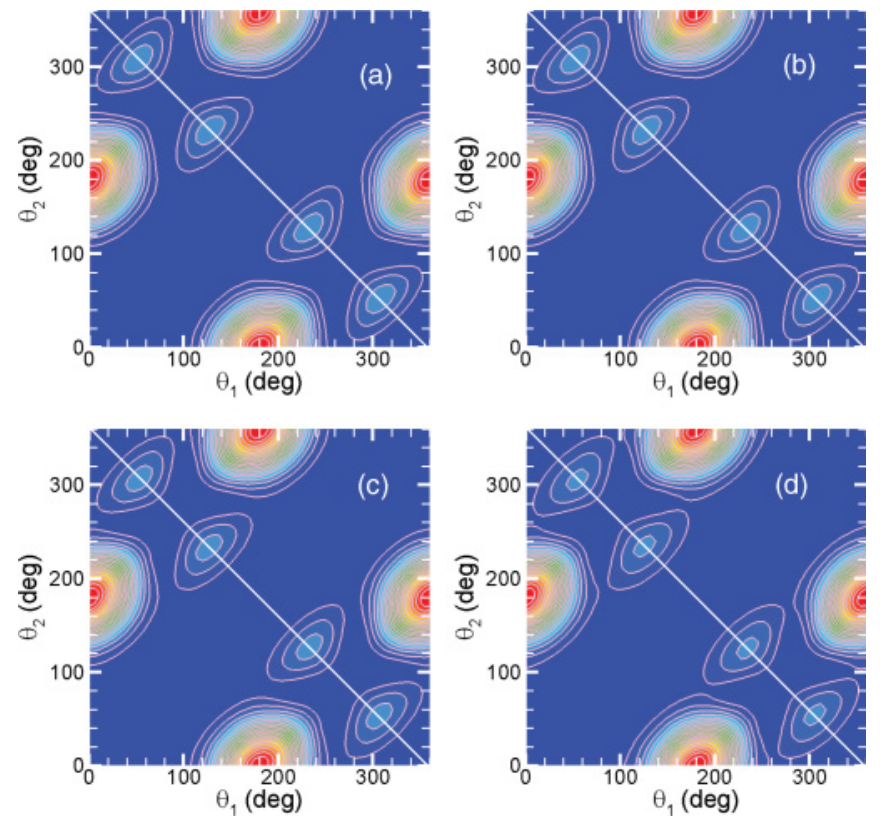

FIG. 3. (Color online) Joint angular distributions $P\left(\theta_{1}, \theta_{2} ; \varepsilon\right)$ for TPDI at $\omega=42 \mathrm{eV}$ for four different electron-energy sharings: (a) $\varepsilon=0.5$, (b) $\varepsilon=0.3$, (c) $\varepsilon=0.1$, and (d) $\varepsilon=0.01$.

as indicated by the major peaks. However, the minor peaks indicate that the two electrons can also be emitted into the same hemisphere with an angle between them, $\theta_{12}$, of about $105^{\circ}$ for $\varepsilon=0.5$. The angle $\theta_{12}$ increases slightly to $107^{\circ}$ and $109^{\circ}$ respectively when $\varepsilon$ is lowered to 0.1 and 0.01 . The similarities in these frames suggests that all the ionized electron pairs, no matter what the energy sharing is, may result from the same underlying correlation mechanism. We note, of course, that electron correlation is necessary for TPDI for photon energies in the range $39.5 \mathrm{eV} \leqslant \hbar \omega \leqslant 54.4 \mathrm{eV}$. Therefore, the question arises whether this general pattern persists for other photon energies in this range for different energy sharing $\varepsilon$. Also, interesting is the question of how this JAD pattern changes for photon energies above this "correlation" range, including energies well above the double-ionization threshold.

Before presenting our results for the JADs at other photon energies, we show in Fig. 4 the angle-integrated doubleionization probability as a function of the energy sharing $\varepsilon$ for various different photon energies $\omega$. Note that the probability for each photon energy is normalized by dividing by the effective interaction time $T_{\text {eff }}$ given in Eq. (12). As can be seen, for $\omega \leqslant 54.4 \mathrm{eV}$, the probability monotonically decreases when $\varepsilon$ changes from 0 to 0.5 . Also, the curves become progressively less flat and more bowl shaped as the photon energy increases from $42 \mathrm{eV}$. For $\omega>54.4 \mathrm{eV}$, two peaks appear, indicating the contribution of the sequential process to double ionization for a sufficiently long pulse. (Note that, for a sufficiently short pulse, the two peaks merge into one [28].) To facilitate the presentation of our results below of the JADs for double ionization at different photon energies, we have selected four representative values of $\varepsilon$ (i.e., $0.5,0.3,0.1$, and 0.01 ). For a given photon energy, we find the JADs at other $\varepsilon$ values are similar to one or more of those for these four $\varepsilon$ values.

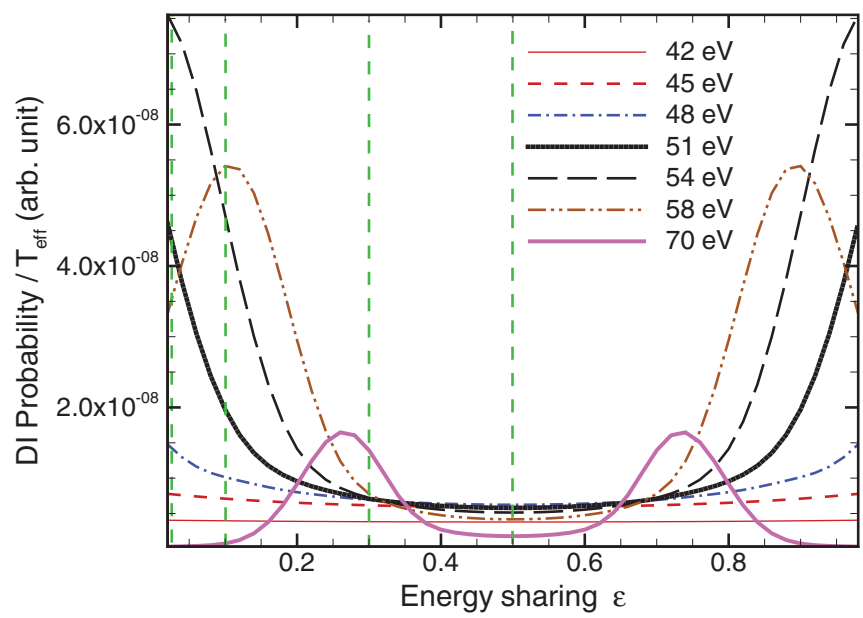

FIG. 4. (Color online) Angle-integrated double-ionization probability, divided by $T_{\text {eff }}$, as a function of the energy sharing $\varepsilon$ [cf. Eq. (16)] for seven different photon energies $\omega$ (indicated in the graph). Vertical dashed lines are drawn through $\varepsilon=0.5,0.3,0.1$, and 0.01 to guide the eye. The joint angular distributions at these values of $\varepsilon$ are shown for various photon energies in subsequent figures.

Finally, a word about our terminology. In what follows, if the JAD has a strong "back-to-back" ionization component, we say that correlations are mainly important in the "intermediate state," (i.e., during the ionization process while the laser field is on). If the dominant feature of the JAD is that the two ionized electrons are ejected in the same hemisphere or along the same axis, we say that correlations are mainly important in the "final state." We shall show also that electron correlations in the "initial state," before the arrival of the laser pulse, have a significant influence on the JAD.

\section{The equal-energy-sharing case}

Most previous studies of angular distributions in TPDI have focused on the angular distribution of one electron with respect to a fixed emission angle of the other electron, especially for the equal-energy-sharing case (i.e., $\varepsilon=0.5$ ). In this case, the two electrons are highly correlated and experience the same effective potential during the process of ionization (i.e., electron correlations in the intermediate state play an important role). Therefore, one may expect that the JADs for different photon energies will be similar. To check whether this expectation is true, we carried out calculations at different photon energies, from the deep nonsequential regime to well above the double-ionization threshold. In Fig. 5, we present the JADs for the case of equal energy sharing at six different photon energies: (a) 45, (b) 51, (c) 54, (d) 58, (e) 70, and (f) $85 \mathrm{eV}$. One sees that the general pattern of the JAD in each frame is very similar to that in Fig. 3(a) for $\omega=42 \mathrm{eV}$; that is, there are four main peaks at the edges and four minor peaks along the line of $\theta_{2}=-\theta_{1}+360^{\circ}$. However, it is important to stress the gradual changes in each frame as the photon energy increases. The first change with increasing photon energy is that the distance between the centers of the first two minor peaks (counted from the left to the right) becomes larger. The second change is that the relative heights of the four minor 

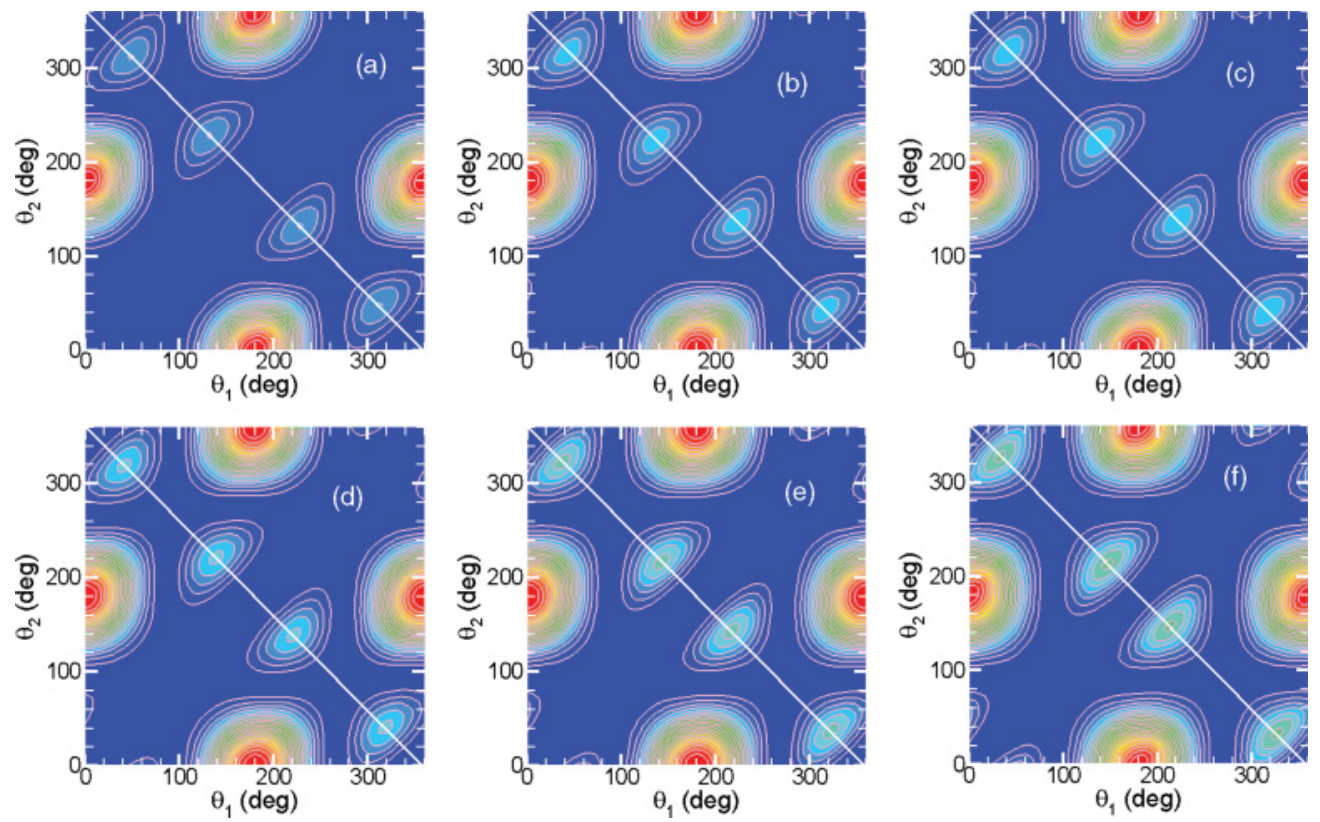

FIG. 5. (Color online) Joint angular distributions $P\left(\theta_{1}, \theta_{2} ; \varepsilon\right)$ for TPDI at equal energy sharing, $\varepsilon=0.5$, for six different photon energies: (a) $\omega=45 \mathrm{eV}$, (b) $\omega=51 \mathrm{eV}$, (c) $\omega=54 \mathrm{eV}$, (d) $\omega=58 \mathrm{eV}$, (e) $\omega=70 \mathrm{eV}$, and (f) $\omega=85 \mathrm{eV}$.

peaks with respect to the four main peaks also become larger. These changes imply that, although electron correlation in the intermediate state is very important at equal energy sharing for any photon energy, the degree of its importance decreases for larger photon energy. In particular, when the two electrons are ejected in the same hemisphere, the angle between them becomes increasingly smaller as the photon energy becomes larger. For example, our calculations show that the angles $\theta_{12}$ between the two electrons emitted in the same hemisphere are about $96^{\circ}, 84^{\circ}$, and $74^{\circ}$, respectively, for photon energies of $\omega=48,54$, and $70 \mathrm{eV}$.

Note that the equal-energy-sharing case $(\varepsilon=0.5)$ for above-threshold ionization [cf. Fig. 5(e) for $\omega=70 \mathrm{eV}$ and Fig. 5(f) for $\omega=85 \mathrm{eV}$ ] corresponds to the "anomalous component" discussed in Ref. [28]. It is known that the two peaks in the energy spectrum (resulting from sequential absorption of two photons by the two electrons) get broadened and merge into a single peak as the pulse duration becomes shorter $[28,42,47]$. Intuitively, one may interpret these results as implying that sequential ionization is gradually replaced by nonsequential double ionization in the case of sufficiently short pulses [20]. Based on their energy distribution results, this interpretation was rejected by Ref. [28]. In their investigation of the anomalous component, Palacios et al. [42] pointed out that their analysis, based on the SDCS, is not able to provide a definitive conclusion regarding the role of electron correlations. Our JAD results in Figs. 5(e) and 5(f), which provide triply differential results, reveal that the two electrons with equal energy sharing undergo a highly correlated doubleionization process. The JAD pattern completely deviates from that of the dipole distribution $\left(\cos ^{2} \theta_{1} \cos ^{2} \theta_{2}\right)$, which is expected in an independent-electron treatment of sequential ionization but is in fact quite similar to that of nonsequential double ionization. So we conclude that electron correlations in the intermediate state govern the double-ionization dynamics at $\varepsilon=0.5$ even for photon energies greater than the doubleionization threshold.

\section{The unequal-energy-sharing case: extreme to moderate}

Different from the equal-energy-sharing case, for $\varepsilon=0.01$, one of the two electrons takes most of the excess energy. In this extreme energy-sharing case, the JADs show different patterns as the photon energy increases. In Fig. 6, we show the JADs for $\varepsilon=0.01$ for six different photon energies: (a) 45 , (b) 48 , (c) 51 , (d) 54 , (e) 70 , and (f) $85 \mathrm{eV}$. One notices that, when $\omega=45$ and $48 \mathrm{eV}$, the JAD pattern looks very similar to the one for $\omega=42 \mathrm{eV}$ given in Fig. 3(d). However, compared with the results presented in Fig. 5 for $\varepsilon=0.5$, the pattern changes drastically as $\omega$ is increased. First, the second and third minor peaks along the $\theta_{2}=-\theta_{1}+360^{\circ}$ line (counting from left to right) gradually shift toward and merge with each other, eventually becoming a single peak when $\omega=70 \mathrm{eV}$. Second, the heights of the minor peaks along the line of $\theta_{2}=-\theta_{1}+$ $360^{\circ}$ rise quickly and become the actual main peaks when $\omega=$ $70 \mathrm{eV}$. As discussed above, for $\varepsilon=0.5$, electron correlations in the intermediate state always dominate at all photon energies. However, for the present extreme energy-sharing case, electron correlations in the final state take the main role as the photon energy increases. The dominant emission pattern of the two electrons changes gradually from the "back-to-back" to the "side-by-side" configuration: the two electrons gradually tend to be ejected in the same hemisphere and even in the same direction along the laser polarization axis.

One may expect that, for the intermediate energy-sharings $\varepsilon=0.3$ and 0.1 , one can observe the changes in the JAD patterns as the photon energy is increased from the nonsequential double-ionization regime to well above the double-ionization threshold. This is indeed the case, as shown in Fig. 7. In this figure, the first row is for $\varepsilon=0.3$ and the second row is for $\varepsilon=0.1$, while the photon energy is taken to be 48,58 , and 

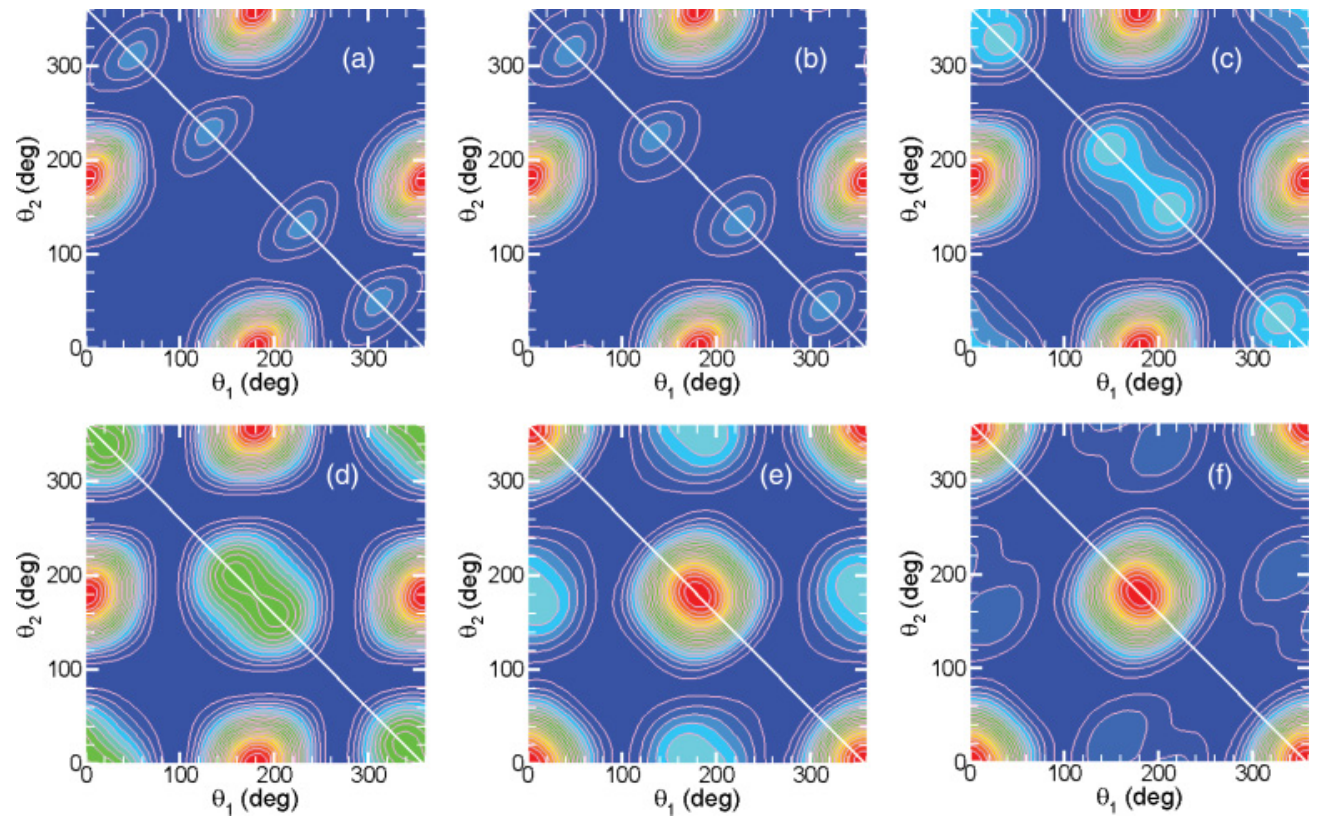

FIG. 6. (Color online) Joint angular distributions $P\left(\theta_{1}, \theta_{2} ; \varepsilon\right)$ for TPDI for extreme energy sharing, $\varepsilon=0.01$, for six different photon energies: (a) $\omega=45 \mathrm{eV}$, (b) $\omega=48 \mathrm{eV}$, (c) $\omega=51 \mathrm{eV}$, (d) $\omega=54 \mathrm{eV}$, (e) $\omega=70 \mathrm{eV}$, and (f) $\omega=85 \mathrm{eV}$.

$70 \mathrm{eV}$ for the left, middle, and right columns, respectively. From these graphs, one can clearly see the trends of the changes in the JAD discussed above. These variations in the JAD clearly demonstrate the different role played by intermediate- and final-state electron correlations. The four minor peaks along $\theta_{2}=-\theta_{1}+360^{\circ}$ gradually merge into two peaks [cf. Figs. 7(c) and 7(e)], showing a characteristic dipole emission pattern. This is because $\varepsilon=0.1(\omega=58 \mathrm{eV})$ and $\varepsilon=0.3(\omega=70 \mathrm{eV})$ almost correspond to the peak positions of the SDCSs in Fig. 4, corresponding to the two electrons being sequentially ejected. In this case, the degree of electron correlations is rather low, especially for the longer-pulse-duration case. In Fig. 7(f), the "side-by-side" emission pattern appears again, indicating the dominance of final-state correlations (i.e., the energy exchange between the two ionized electrons). We conclude that, as the photon energy increases above the second-ionization threshold, the final-state electron correlations gradually become more important than electron correlations in the intermediate state, and the smaller the energy sharing $\varepsilon$, the faster this will occur.
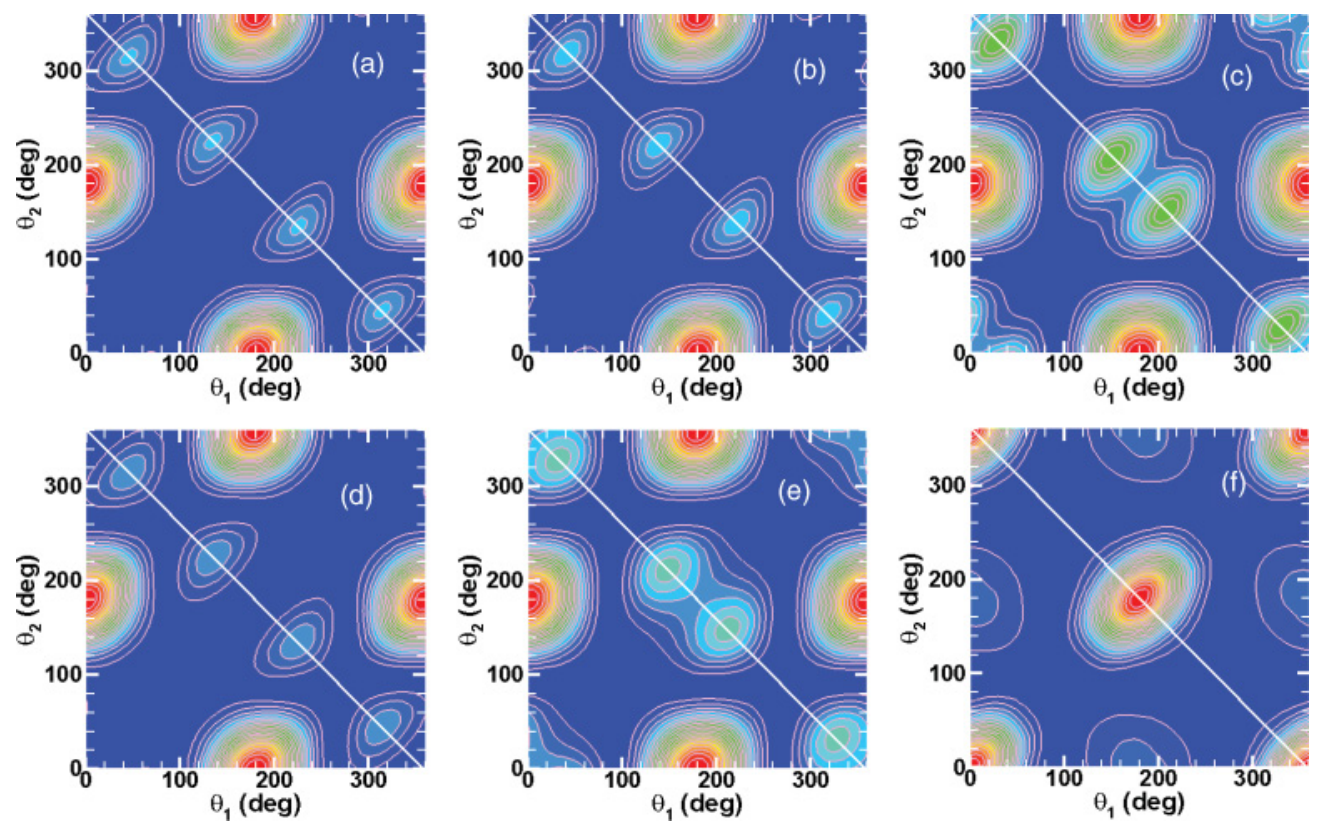

FIG. 7. (Color online) Joint angular distributions $P\left(\theta_{1}, \theta_{2} ; \varepsilon\right)$ for TPDI for the two energy sharings $\varepsilon=0.3$ [(a)-(c)] and $\varepsilon=0.1$ [(d)-(f)] and for the three photon energies $\omega=48 \mathrm{eV}$ [(a),(d)], $\omega=58 \mathrm{eV}$ [(b), (e)], and $\omega=70 \mathrm{eV}$ [(c), (f)]. 

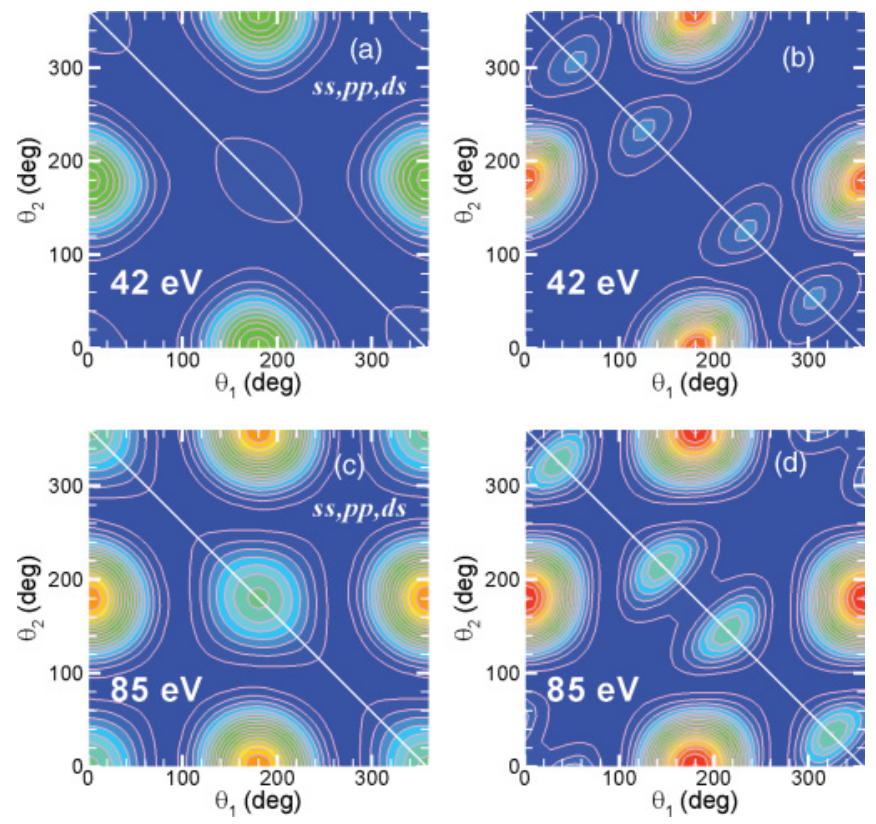

FIG. 8. (Color online) JAD for $\omega=42[(\mathrm{a}),(\mathrm{b})]$ and $\omega=85 \mathrm{eV}$ [(c), (d)]. In (a) and (c), only the $s s, p p$, and $d s$ final-state angular momentum components are included, while all angular momentum components (up to the limits of our calculation) are included in (b) and (d).

\section{Initial-state correlation effects on JAD}

For both the nonsequential and sequential ionization regimes for $\varepsilon=0.5$, we can further identify the importance of electron correlations in the initial state. Owing to electron correlations, the ground state of $\mathrm{He}$ can be expanded in configurations of the form of $\left|1 s^{2}\right\rangle=a^{(0)}|s\rangle|s\rangle+a^{(1)}|p\rangle|p\rangle+$ $a^{(2)}|d\rangle|d\rangle+\cdots$, with $\left|a^{(0)}\right| \gtrsim 10\left|a^{(1)}\right|$. For double ionization from the dominant $|s\rangle|s\rangle$ component, the resulting ionized electrons are restricted by electric dipole selection rules for a two-photon process to the following partial waves: $|s\rangle|s\rangle$, $|p\rangle|p\rangle$, or $|s\rangle|d\rangle(|d\rangle|s\rangle)$. In Fig. 8(a), we present the JAD corresponding to the inclusion of only the $|s\rangle|s\rangle,|p\rangle|p\rangle$, or $|s\rangle|d\rangle(|d\rangle|s\rangle)$ partial wave components at $\varepsilon=0.5$ for $\omega=42 \mathrm{eV}$. The two electrons tend to be ejected back to back, but no minor peaks are found along the diagonal line defined by $\theta_{2}=-\theta_{1}+360^{\circ}$. However, if all partial wave components (up to the limit of our calculation) are included [cf. Fig. 8(b)], the JAD pattern changes. First, the four minor peaks along $\theta_{2}=-\theta_{1}+360^{\circ}$ emerge. Second, the peaks corresponding to back-to-back emission get much stronger compared to those in Fig. 8(a). The contributions of these higher $l_{1}, l_{2}$ partial waves come from the components $|p\rangle|p\rangle,|d\rangle|d\rangle$, etc., included in the correlated initial state. Similar observations apply for the case of a photon energy well above the double-ionization threshold and equal energy sharing, as shown in Figs. 8(c) and 8(d) for $\omega=85 \mathrm{eV}$. These results show that electron correlation in the initial state may also play an important role in TPDI.

\section{CONCLUSIONS}

By directly solving the time-dependent, full-dimensional, two-electron Schrödinger equation for He in the field of a laser pulse, we investigate the two-photon double-ionization process for the case of xuv laser pulses in both the nonsequential and the sequential regimes. We have carried out a systematic analysis of the joint angular distribution of the two ionized electrons as a means to elucidate the role of electron correlations in TPDI. In direct (nonsequential) double ionization, the back-to-back emission pattern always dominates, indicating the importance of electron correlations in the intermediate state (i.e., during the interaction of the electrons with the laser pulse). Such a pattern in the JAD is found to be a general one for any energy sharing for photon energies less than $54.4 \mathrm{eV}$. This distribution pattern thus serves as a hallmark of electron correlation in the intermediate state. In the sequential double-ionization regime, if the two electrons share the excess energy equally, the dominant correlation mechanism is similar to that of the nonsequential double-ionization regime. However, for extremely unequal energy sharing, the Coulomb repulsion between the electrons in the final state (after the end of the laser pulse) becomes the dominant electron correlation effect. Finally, for both the nonsequential and sequential regimes, initial-state electron correlation effects have been demonstrated in the JAD patterns. Namely, an uncorrelated initial state produces only a few angular momentum partial waves in the TPDI process, whereas a correlated ground state produces a richer number of partial waves. The number of partial waves has been shown to greatly affect the pattern of the JAD. These various investigations have thus demonstrated the value of the JAD as a means of elucidating two-electron dynamics.

\section{ACKNOWLEDGMENTS}

This work was supported in part by the National Natural Science Foundation of China under Grants No. 10974007, No. 11174016, and No. 10821062, by the US Department of Energy, Office of Science, Division of Chemical Sciences, Geosciences, and Biosciences, under Grant No. DE-FG0396ER14646, and by a Grant-in-Aid from the Japan Society for the Promotion Science (JSPS). L.-Y.P. thanks Johannes Feist for sending us his data in numerical form for our comparisons in Fig. 2.
[1] D. N. Fittinghoff, P. R. Bolton, B. Chang, and K. C. Kulander, Phys. Rev. Lett. 69, 2642 (1992).

[2] B. Walker, B. Sheehy, L. F. DiMauro, P. Agostini, K. J. Schafer, and K. C. Kulander, Phys. Rev. Lett. 73, 1227 (1994).

[3] Th. Weber, H. Giessen, M. Weckenbrock, G. Urbasch, A. Staudte, L. Spielberger, O. Jagutzki, V. Mergel, M. Vollmer, and R. Dörner, Nature (London) 405, 658 (2000).
[4] A. Staudte et al., Phys. Rev. Lett. 99, 263002 (2007).

[5] A. Rudenko, V. L. B. de Jesus, Th. Ergler, K. Zrost, B. Feuerstein, C. D. Schröter, R. Moshammer, and J. Ullrich, Phys. Rev. Lett. 99, 263003 (2007).

[6] X. Liu and C. Figueira de Morisson Faria, Phys. Rev. Lett. 92, 133006 (2004).

[7] X. Liu et al., Phys. Rev. Lett. 93, 263001 (2004). 
[8] Y. Liu, S. Tschuch, A. Rudenko, M. Dürr, M. Siegel, U. Morgner, R. Moshammer, and J. Ullrich, Phys. Rev. Lett. 101, 053001 (2008).

[9] Y. Liu et al., Phys. Rev. Lett. 104, 173002 (2010).

[10] W. Ackermann et al., Nat. Photonics 1, 336 (2007).

[11] T. Shintake et al., Nat. Photonics 2, 555 (2008).

[12] B. McNeil, Nat. Photonics 3, 375 (2009).

[13] G. Sansone et al., Science 314, 443 (2006).

[14] E. Goulielmakis et al., Science 320, 1614 (2008).

[15] J. Feist, R. Pazourek, S. Nagele, E. Persson, B. I. Schneider, L. A. Collins, and J. Burgdörfer, J. Phys. B 42, 134014 (2009).

[16] E. Foumouo, A. Hamido, Ph. Antoine, B. Piraux, H. Bachau, and R. Shakeshaft, J. Phys. B 43, 091001 (2010).

[17] P. Lambropoulos, G. M. Nikolopoulos, and K. G. Papamihail, Phys. Rev. A 83, 021407 (2011).

[18] P. B. Corkum, Phys. Rev. Lett. 71, 1994 (1993).

[19] M. S. Pindzola and F. Robicheaux, J. Phys. B 31, L823 (1998).

[20] J. S. Parker, L. R. Moore, K. J. Meharg, D. Dundas, and K. T. Taylor, J. Phys. B 34, L69 (2001).

[21] L. A. A. Nikolopoulos and P. Lambropoulos, J. Phys. B 34, 545 (2001); 40, 1347 (2007).

[22] M. G. Makris, L. A. A. Nikolopoulos, and P. Lambropoulos, Europhys. Lett. 54, 722 (2001).

[23] J. Colgan and M. S. Pindzola, Phys. Rev. Lett. 88, 173002 (2002).

[24] L. Feng and H. W. van der Hart, J. Phys. B 36, L1 (2003).

[25] S. Laulan and H. Bachau, Phys. Rev. A 68, 013409 (2003).

[26] B. Piraux, J. Bauer, S. Laulan, and H. Bachau, Eur. Phys. J. D 26, 7 (2003).

[27] S. X. Hu, J. Colgan, and L. A. Collins, J. Phys. B 38, L35 (2005).

[28] K. L. Ishikawa and K. Midorikawa, Phys. Rev. A 72, 013407 (2005).

[29] I. F. Barna, J. Wang, and J. Burgdörfer, Phys. Rev. A 73, 023402 (2006).

[30] S. X. Hu and L. A. Collins, Phys. Rev. Lett. 96, 073004 (2006).

[31] A. Y. Istomin, E. A. Pronin, N. L. Manakov, S. I. Marmo, and A. F. Starace, Phys. Rev. Lett. 97, 123002 (2006).

[32] E. Foumouo, G. Lagmago Kamta, G. Edah, and B. Piraux, Phys. Rev. A 74, 063409 (2006).

[33] I. A. Ivanov and A. S. Kheifets, Phys. Rev. A 75, 033411 (2007).

[34] E. A. Pronin, N. L. Manakov, S. I. Marmo, and A. F. Starace, J. Phys. B 40, 3115 (2007).

[35] D. A. Horner, F. Morales, T. N. Rescigno, F. Martín, and C. W. McCurdy, Phys. Rev. A 76, 030701 (2007).

[36] E. Foumouo, Ph. Antoine, B. Piraux, L. Malegat, H. Bachau, and R. Shakeshaft, J. Phys. B 41, 051001 (2008).

[37] A. Palacios, T. N. Rescigno, and C. W. McCurdy, Phys. Rev. A 77, 032716 (2008).

[38] J. Feist, S. Nagele, R. Pazourek, E. Persson, B. I. Schneider, L. A. Collins, and J. Burgdörfer, Phys. Rev. A 77, 043420 (2008).

[39] X. Guan, K. Bartschat, and B. I. Schneider, Phys. Rev. A 77, 043421 (2008).

[40] D. A. Horner, C. W. McCurdy, and T. N. Rescigno, Phys. Rev. A 78, 043416 (2008).
[41] I. A. Ivanov and A. S. Kheifets, Phys. Rev. A 79, 023409 (2009).

[42] A. Palacios, T. N. Rescigno, and C. W. McCurdy, Phys. Rev. A 79, 033402 (2009).

[43] J. Feist, S. Nagele, R. Pazourek, E. Persson, B. I. Schneider, L. A. Collins, and J. Burgdörfer, Phys. Rev. Lett. 103, 063002 (2009).

[44] A. Palacios, T. N. Rescigno, and C. W. McCurdy, Phys. Rev. Lett. 103, 253001 (2009).

[45] D. A. Horner, T. N. Rescigno, and C. W. McCurdy, Phys. Rev. A 81, 023410 (2010).

[46] Z. Zhang, L.-Y. Peng, Q. Gong, and T. Morishita, Opt. Express 18, 8976 (2010).

[47] A. Palacios, D. A. Horner, T. N. Rescigno, and C. W. McCurdy, J. Phys. B 43, 194003 (2010).

[48] H. Bachau, Phys. Rev. A 83, 033403 (2011).

[49] R. Pazourek, J. Feist, S. Nagele, E. Persson, B. I. Schneider, L. A. Collins, and J. Burgdörfer, Phys. Rev. A 83, 053418 (2011).

[50] H. Hasegawa, E. J. Takahashi, Y. Nabekawa, K. L. Ishikawa, and K. Midorikawa, Phys. Rev. A 71, 023407 (2005).

[51] A. A. Sorokin, M. Wellhöfer, S. V. Bobashev, K. Tiedtke, and M. Richter, Phys. Rev. A 75, 051402 (2007).

[52] R. Moshammer et al., Phys. Rev. Lett. 98, 203001 (2007).

[53] A. Rudenko et al., Phys. Rev. Lett. 101, 073003 (2008).

[54] S. Fritzsche, A. N. Grum-Grzhimailo, E. V. Gryzlova, and N. M. Kabachnik, J. Phys. B 42, 145602 (2009).

[55] L. B. Madsen, L. A. A. Nikolopoulos, T. K. Kjeldsen, and J. Fernández, Phys. Rev. A 76, 063407 (2007).

[56] T. N. Rescigno and C. W. McCurdy, Phys. Rev. A 62, 032706 (2000).

[57] L. Tao, C. W. McCurdy, and T. N. Rescigno, Phys. Rev. A 82, 023423 (2010).

[58] F. L. Yip, C. W. McCurdy, and T. N. Rescigno, Phys. Rev. A 81, 063419 (2010).

[59] C. W. McCurdy, M. Baertschy, and T. N. Rescigno, J. Phys. B 37, R137 (2004).

[60] L.-Y. Peng, J. F. McCann, D. Dundas, K. T. Taylor, and I. D. Williams, J. Chem. Phys. 120, 10046 (2004).

[61] L.-Y. Peng and A. F. Starace, J. Chem. Phys. 125, 154311 (2006).

[62] L. Malegat, H. Bachau, A. Hamido, and B. Piraux, J. Phys. B 43, 245601 (2010).

[63] A. F. Starace, Theory of Atomic Photoionization, Handbuch der Physik, edited by W. Mehlhorn (Springer-Verlag, Berlin, 1982), Vol. 31, pp. 1-121 (cf. pp. 7, 14).

[64] A. Scrinzi and B. Piraux, Phys. Rev. A 58, 1310 (1998).

[65] Springer Handbook of Atomic, Molecular, and Optical Physics, edited by G. W. F. Drake (Springer, New York, 2006), pp. 205, 206.

[66] J. S. Briggs and V. Schmidt, J. Phys. B 33, R1 (2000).

[67] T. Schneider, P. L. Chocian, and J.-M. Rost, Phys. Rev. Lett. 89, 073002 (2002).

[68] R. Dörner et al., Phys. Rev. A 57, 1074 (1998).

[69] J. A. R. Samson, W. C. Stolte, Z.-X. He, J. N. Cutler, Y. Lu, and R. J. Bartlett, Phys. Rev. A 57, 1906 (1998). 